\title{
Beam dynamics of a new low emittance third generation synchrotron light source facility
}

\author{
H. Ghasem, ${ }^{1,2, *}$ E. Ahmadi, ${ }^{2,3}$ F. Saeidi, ${ }^{2,4}$ and K. Sarhadi ${ }^{2}$ \\ ${ }^{1}$ School of Particles and Accelerators, Institute for Research in Fundamental Sciences (IPM), Tehran, Iran \\ ${ }^{2}$ Iranian Light Source Facility (ILSF), Institute for Research in Fundamental Sciences (IPM), Tehran, Iran \\ ${ }^{3}$ Department of Physics, Iran University of Science and Technology, Tehran, Iran \\ ${ }^{4}$ Department of Physics, Faculty of Science, Arak University, Arak, Iran
}

(Received 9 November 2014; published 26 March 2015)

\begin{abstract}
The Iranian Light Source Facility (ILSF) is a new $3 \mathrm{GeV}$ third generation synchrotron light source facility which is in the design stage. As the main radiation source, design of the ILSF storage ring emphasizes an ultralow electron beam emittance, great brightness, stability and reliability. The storage ring is based on a five-bend achromat lattice providing an ultralow horizontal beam emittance of $0.48 \mathrm{~nm} \mathrm{rad}$. In this paper, we present the design feature of the ILSF storage ring, give the linear and nonlinear dynamic properties of the lattice and discuss the related beam dynamic specifications.
\end{abstract}

DOI: 10.1103/PhysRevSTAB.18.030710

PACS numbers: 29.20.db, 41.75.Ht, 42.72.-g

\section{INTRODUCTION}

A synchrotron light source facility is well known for the community of the researchers as an essential laboratory that provides high bright synchrotron radiation for their experiments in different disciplines. Due to the numerous applications of the synchrotron radiation, many synchrotron light sources are under operation or construction around the world and several countries are planning to have their own light source facility. However, the Middle East, as a large portion of the world, is still poor in such modern facilities. After SESAME which has been dedicated by UNESCO [1], Turkey and Armenia are involved with their accelerator projects [2,3]. In parallel, the government of Iran is convinced to build an intermediate energy synchrotron light source called as Iranian Light Source Facility (ILSF) with several beam lines to cover the submitted user's request proposals [4,5]. The ILSF project is the first Iranian synchrotron radiation source which will be built in the city of Qazvin located $150 \mathrm{~km}$ west of Tehran. Based on the ILSF strategy, various requirements of the modern synchrotron radiation sources cannot be totally fulfilled at this facility but it will provide super bright synchrotron radiation required for the cutting-edge science in several fields and will serve as a significant impetus for the multidisciplinary researches.

Design of the ILSF accelerators was commenced in June 2010. Regarding the proposed budget and user's requirements, several types of lattice with different configurations of

\footnotetext{
Corresponding author. ghasem@ipm.ir

Published by the American Physical Society under the terms of the Creative Commons Attribution 3.0 License. Further distribution of this work must maintain attribution to the author(s) and the published article's title, journal citation, and DOI.
}

the magnets have been explored for the storage ring [6-8]. The lattice design studies and the experiences of R\&D fabricated magnet prototypes with home industries revealed that use of the local available weak field material (low carbon stainless steel, ST14, standard number 1.0338) for the magnets in comparison with the high field one would be beneficial for the ILSF accelerators in several points of view [9-11]. Lower required rf power, less needed power supply and cooling systems are some of the benefits which all result to save energy and minimize operation cost. However, strong quadrupoles are required in the ultralow beam emittance lattice and accordingly strong sextupoles are needed to solve the lattice nonlinear problems. Therefore the magnets have been designed with small apertures to provide highest field strength. It causes a big impact on the vacuum system design. Because of the small aperture of the weak magnets and as a consequence for the ultralow emittance beam, a low emittance booster is designed to place at the same tunnel as the storage ring to assure an efficient injection process and to reduce the construction costs. Moreover, the single role magnets are employed in the lattice design which result in low fabrication cost with local industries in comparison with the high field one, easy alignment in the ring and less sensitivity of lattice to the errors. Although the radiated photon beam critical energy from the low field dipoles would be useful for the several experiments in the soft $\mathrm{x}$-ray region, the designed lattice can provide the required high energy hard $\mathrm{x}$-ray photon beam with the use of the super-bend magnet which additionally helps emittance reduction (see Sec. VI).

This paper starts with the detailed descriptions of the optimized ILSF storage ring lattice and then is followed by the expected nonlinear dynamic performance. Effects of the errors on the closed orbit and the optimized global correction scheme have been given next. Super bright high energy radiation from the super-bend magnet and its corresponding beam dynamic issues will be explained. 


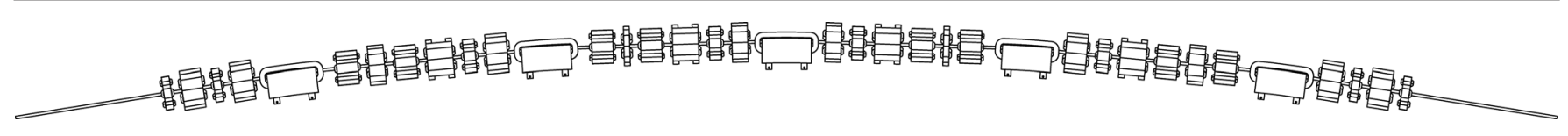

FIG. 1. Top view of one super period of the ILSF storage ring lattice [24].

Section VII describes the ILSF operation scenarios, demonstrates radiation performance and gives the effects of insertion devices (IDs) on the electron beam dynamics. Lifetime calculation including intrabeam scattering (IBS) effects and injection procedure are the last issues. It should be noted that the OPA [12], ELEGANT [13], MADX [14], BETA [15] simulation codes are used for the design, linear/ nonlinear optics optimization, particle tracking and the SPECTRA [16] code is employed to study radiation properties.

\section{LATTICE}

Several intensive efforts have been performed in the design of the third generation synchrotron light sources around the world to meet future demands of the users for having super bright radiation from ultralow electron beam emittance [17-21]. The beam emittance is defined by the structure of magnets in the lattice. In an isomagnetic storage ring lattice, it is proportional directly to the square of beam energy and inversely to the third power of number of dipoles. Horizon of the ultralow emittance storage rings is based on the multibend achromat (MBA) lattice structure which improves the brightness 2 to 3 orders of magnitude higher than nowadays synchrotron radiation light sources. Similar to the recently designed [22,23] and upgraded $[20,21]$ light sources, the ILSF storage ring lattice is designed based on multidipole per super period to provide an ultralow beam emittance and long enough straight sections which are the main concerns from the user's point of view. In order to avoid large storage ring circumference and to have a large number of beam lines, the designed lattice is optimized to be as compact as possible. The ILSF storage ring circumference is $528 \mathrm{~m}$. It is composed of 20 super periods and provides 20 straight sections each with the length of $5.11 \mathrm{~m}$. One of them will be occupied

TABLE I. Main ILSF storage ring parameters. The subscripts $x, y$ and $s$ indicate horizontal, vertical and longitudinal directions respectively.

\begin{tabular}{|c|c|c|c|}
\hline Parameter & Symbol & Unit & Value \\
\hline Energy & $E$ & $\mathrm{GeV}$ & 3 \\
\hline Maximum beam current & $I_{b}$ & $\mathrm{~mA}$ & 400 \\
\hline Circumference & C & $\mathrm{m}$ & 528 \\
\hline Lattice structure & $\cdots$ & $\cdots$ & $5 \mathrm{BA}$ \\
\hline Number of super period & $\ldots$ & $\cdots$ & 20 \\
\hline Length of straight sections & $L$ & $\mathrm{~m}$ & 5.110 \\
\hline Natural emittance & $\varepsilon$ & pm rad & 476.621 \\
\hline Betatron tune & $Q_{x} / Q_{y}$ & $-/-$ & $43.283 / 14.255$ \\
\hline Natural chromaticity & $\xi_{x} / \xi_{y}$ & $-/-$ & $-99.432 /-52.825$ \\
\hline First order momentum compaction factor & $\alpha_{c}$ & $-/-$ & $1.526 \times 10^{-4}$ \\
\hline Second order momentum compaction factor & $\alpha_{c}^{2 \text { nd }}$ & $-/-$ & $4.443 \times 10^{-4}$ \\
\hline Natural energy spread & $\delta$ & $-/-$ & $7.028 \times 10^{-4}$ \\
\hline Damping times & $\tau_{x} / \tau_{y} / \tau_{s}$ & $\mathrm{~ms} / \mathrm{ms} / \mathrm{ms}$ & $19.709 / 19.716 / 9.859$ \\
\hline Natural energy loss per turn & $U_{0}$ & $\mathrm{keV}$ & 535.975 \\
\hline Revolution time & $\tau_{\operatorname{Rev}}$ & $\mu \mathrm{s}$ & 1.760 \\
\hline Radiation power from dipoles @ 400 mA & $P_{\mathrm{Rad}}$ & $\mathrm{kW}$ & 214.390 \\
\hline rf frequency & $f_{\mathrm{RF}}$ & $\mathrm{MHz}$ & 500 \\
\hline Harmonic number & $h$ & $\ldots$ & 880 \\
\hline Radiation integral, $I_{1}$ & $I_{1}$ & $\mathrm{~m}$ & $8.059 \times 10^{-2}$ \\
\hline Radiation integral, $I_{2}$ & $I_{2}$ & $1 / \mathrm{m}$ & $4.699 \times 10^{-1}$ \\
\hline Radiation integral, $I_{3}$ & $I_{3}$ & $1 / \mathrm{m}^{2}$ & $3.515 \times 10^{-2}$ \\
\hline Radiation integral, $I_{4}$ & $I_{4}$ & $1 / \mathrm{m}$ & $-1.547 \times 10^{-4}$ \\
\hline Radiation integral, $I_{5}$ & $I_{5}$ & $1 / \mathrm{m}$ & $1.697 \times 10^{-5}$ \\
\hline Average of beta function & $\left\langle\beta_{x}\right\rangle /\left\langle\beta_{y}\right\rangle$ & $\mathrm{m} / \mathrm{m}$ & $7.583 / 8.055$ \\
\hline Transverse beam size at center of straight section & $\sigma_{x} / \sigma_{y}$ & $\mu \mathrm{m} / \mu \mathrm{m}$ & $92.988 / 3.702$ \\
\hline Minimum/maximum horizontal beta function & $\beta_{x \operatorname{Min}} / \beta_{x \operatorname{Max}}$ & $\mathrm{m} / \mathrm{m}$ & $0.791 / 18.607$ \\
\hline Minimum/maximum vertical beta function & $\beta_{y \operatorname{Min}} / \beta_{y \operatorname{Max}}$ & $\mathrm{m} / \mathrm{m}$ & $2.829 / 19.321$ \\
\hline Minimum/maximum horizontal dispersion function & $\eta_{x \operatorname{Min}} / \eta_{x \operatorname{Max}}$ & $\mathrm{cm} / \mathrm{cm}$ & $0.000 / 11.062$ \\
\hline
\end{tabular}




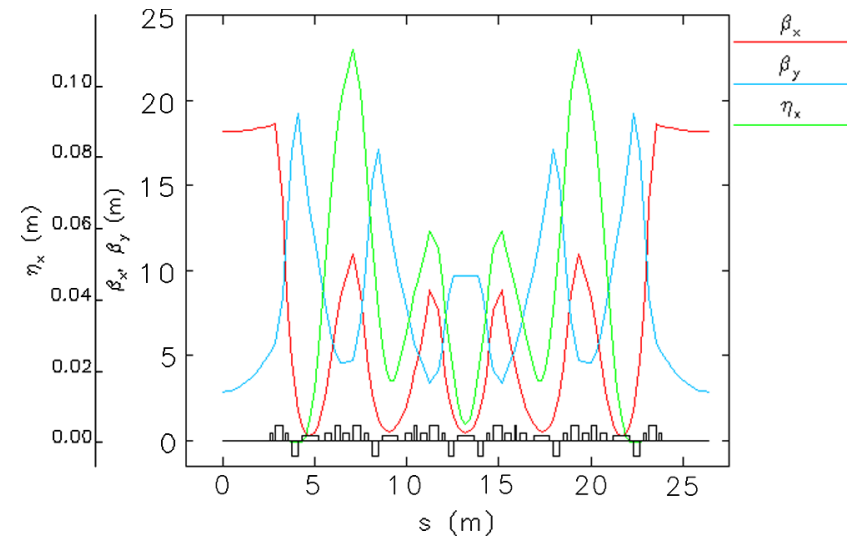

FIG. 2. The optical functions in a super period of the storage ring.

with the injection equipment, two of them are reserved for the rf cavities; the remaining straight sections are considered for installation of the insertion devices with the length up to $4 \mathrm{~m}$. A mechanical drawing of one super period of the storage ring lattice is displayed in Fig. 1. It is composed of five dipoles and a total of 100 in the whole storage ring. Each dipole includes no field gradient, bending field of $0.75 \mathrm{~T}$, length of $0.84 \mathrm{~m}$ and bends the beam 3.60 degrees. Focusing is performed with the use of 16 quadrupoles grouped in eight families. There are totally 320 quadrupoles with maximum strength of $25 \mathrm{~T} / \mathrm{m}$ and pole radius of $26 \mathrm{~mm}$. The main ring parameters are given in Table I and the optical functions in a super period of the storage ring are shown in Fig. 2. With use of such relaxed weak magnets in the lattice design, an extraordinary natural emittance of about $0.48 \mathrm{~nm}$ rad is achieved. The lattice has been tuned to provide high horizontal and low vertical beta functions at the straight sections which are required to have an efficient beam injection and to minimize the impact of the insertion devices, respectively. As depicted in Fig. 2, the dispersion function is kept to zero at the straight section and reaches the maximum of $11 \mathrm{~cm}$ at the arc section.

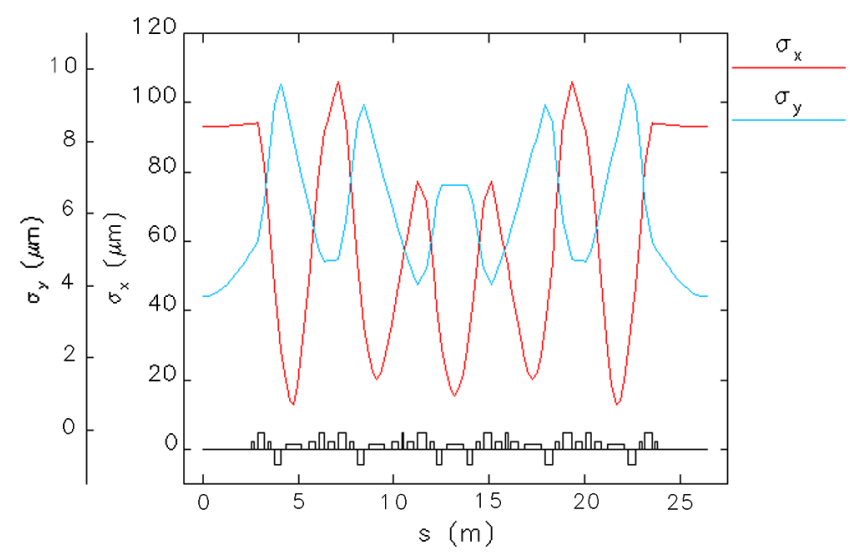

FIG. 3. The beam size in a super period of the storage ring.
The photon diffraction limit $\left(\varepsilon_{\mathrm{DL}}=\lambda / 4 \pi\right)$ for the emitted wavelength of $1 \AA$ is $7.96 \mathrm{pm}$ rad which is 1.67 times the vertical beam emittance at $1 \%$ coupling (4.8 pm rad). This corresponds to the vertical beam size of $4.74 \mu \mathrm{m}$ at the center of the straight section. Beam size in a super period of the storage ring is shown in Fig. 3. It indicates that the vertical beam size at the straight sections is below the diffraction limit for the radiation wavelength of $1 \AA$.

\section{NONLINEAR DYNAMICS}

To suppress transverse head-tail instabilities and to avoid large tune spread of the off energy electrons, the natural horizontal/vertical chromaticity is corrected close to a positive value $(2.0 / 2.2)$ by the use of 16 sextupole magnets grouped in eight families with maximum strength of $1200\left(\mathrm{~T} / \mathrm{m}^{2}\right)$ and pole radius of $26 \mathrm{~mm}$. We aimed to place the sextupoles at the suitable locations with the proper phase advances in the lattice to minimize their nonlinear effects and to avoid the dangerous resonances. For a given tune point of the ILSF compact low emittance lattice, the chromatic and geometric driving terms and their parametric dependency on sextupole strengths are optimized using OPA [12] code and then dynamic aperture is evaluated by the numerical particle tracking. This procedure is repeated simultaneously several times for different tune points and various sextupole configurations to obtain the required dynamic aperture. The optimum dynamic aperture of the on momentum electrons and the electrons with $\pm 4 \%$ energy deviation at the center of the straight section is depicted in Fig. 4 for the ideal ring without errors. Obtaining such large dynamic aperture

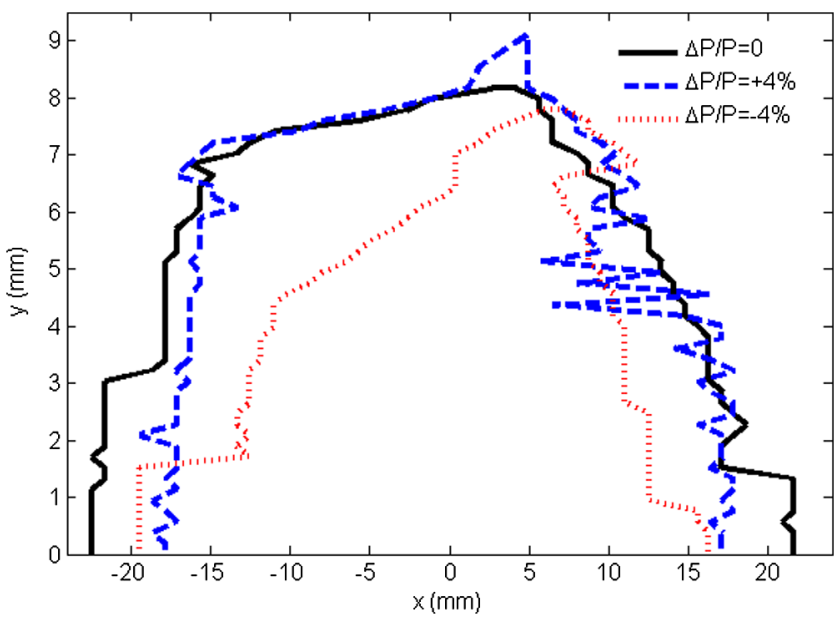

FIG. 4. The dynamic aperture at the center of the straight section. The horizontal/vertical chromaticity is corrected to 2.0/2.2. The particle tracking has been done for 5000 turns passing through the storage ring. 


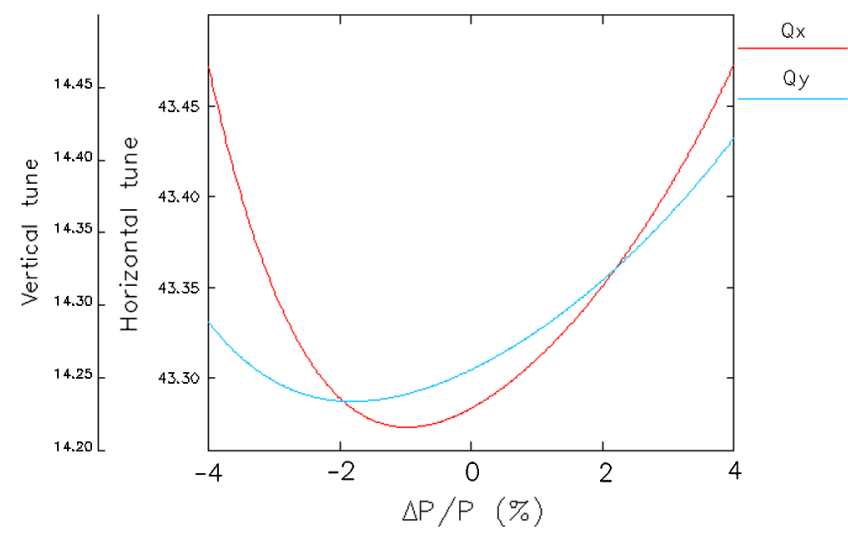

FIG. 5. Shift of transverse tune due to $\pm 4 \%$ energy errors.

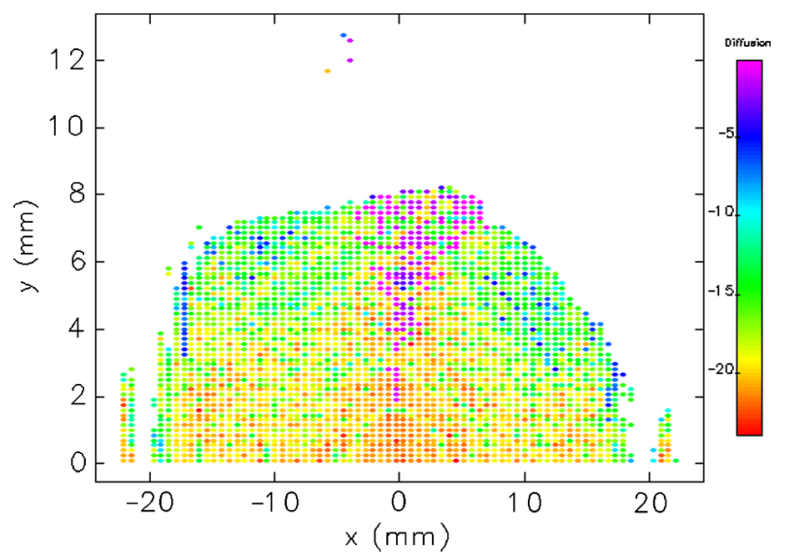

FIG. 6. Frequency map analysis of the bare lattice. The on energy electrons have been tracked 5000 turns through the storage ring. The colors indicate diffused transverse fractional tunes, $\log \sqrt{\Delta Q_{x}^{2}+\Delta Q_{y}^{2}}$.

which is one of the main challenges in the ultralow emittance storage rings is required by the good beam injection efficiency and having a longer Touschek lifetime.

The smooth shift of the tune point due to the energy deviation is depicted in Fig. 5. Low values of the tune spread reveal that the present sextupole arrangement was a good starting point to perform lattice nonlinear optimization and indicate a promising behavior of the tune point up to $\pm 4 \%$ energy deviations.

A diffusion map of the dynamic aperture is depicted in Fig. 6 and corresponding tune diagrams are given in Fig. 7. It should be mentioned that this is an ongoing optimization work.

The small multipole errors and nonlinear fields in the lattice magnetic elements produce high order resonances at the aperture boundary and always result to shrink the dynamic aperture. To study the effects of the higher multipoles, systematic and random components of the multipoles given in Table II have been utilized in our simulation and then particle tracking has been done for 5000 turns. The dynamic aperture of the on/off momentum electrons in the presence of higher multipoles of the magnets is shown in Fig. 8.

\section{CLOSED ORBIT}

There are many sources of errors in the high intensity electron storage rings that cause closed orbit distortion (COD). The error in the field of the magnets caused by the fabrication process is one of them. Girders deformation, roll and displacement of the magnetic elements with respect to the girders are also the other sources of the COD. Misalignment of the quadrupoles has the most sever effects and the out coming dipole field is relative to both gradient and misalignment errors. In order to study sensitivity of the closed orbit to the errors and to determine the optimum global correction scheme, various expected seeds of the errors given in Table III are imposed randomly into the ring. We employed 360 beam position monitors (BPMs) to monitor the beam coordinates and orbit study. They have been placed at the crucial points in the lattice, near to the quadrupoles and sextupoles for COD minimization and feed-down effects reduction respectively. We additionally placed two BPMs at both ends of each straight section to improve stability control of the emitted synchrotron light from the IDs. In order to correct the distorted orbit,
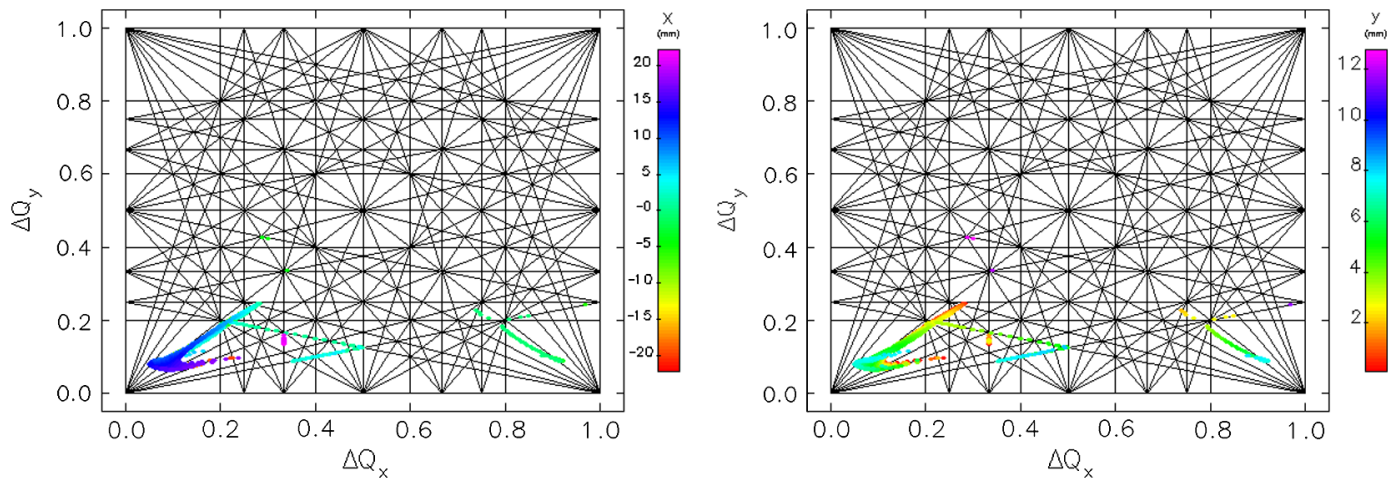

FIG. 7. Variation of the fraction of the tune point due to the amplitude in tune diagram with 5th order of resonance lines. The colors indicate (left) horizontal and (right) vertical amplitudes. 
TABLE II. Systematic and random multipole components of the magnets [11]. The field expansion is assumed to be $B_{y}=\sum_{n=1}^{n=\infty} B_{n}$, where $B_{n}=x^{n-1} /(n-1) !\left(\partial^{n-1} B / \partial x^{n-1}\right)$ and $n$ is the order of the magnet.

\begin{tabular}{|c|c|c|c|c|}
\hline & Order & Multipole & Systematic & Random \\
\hline \multirow{8}{*}{$\begin{array}{l}\text { Dipole } B_{n} / B_{0} \\
\quad @ 13.5 \mathrm{~mm}\end{array}$} & 2 & 4-pole & 0.00 & $1.00 \times 10^{-6}$ \\
\hline & 3 & 6-pole & $1.50 \times 10^{-4}$ & $1.00 \times 10^{-6}$ \\
\hline & 4 & 8-pole & 0.00 & $1.00 \times 10^{-6}$ \\
\hline & 5 & 10-pole & $4.75 \times 10^{-5}$ & $1.00 \times 10^{-6}$ \\
\hline & 6 & 12-pole & 0.00 & $1.00 \times 10^{-6}$ \\
\hline & 7 & 14-pole & $-8.25 \times 10^{-5}$ & $1.00 \times 10^{-6}$ \\
\hline & 8 & 16-pole & 0.00 & $1.00 \times 10^{-6}$ \\
\hline & 9 & 18-pole & $-6.00 \times 10^{-5}$ & $1.00 \times 10^{-6}$ \\
\hline \multirow{17}{*}{$\begin{array}{l}\text { Quadrupole } B_{n} / B_{1} \\
\text { @ } 13.5 \mathrm{~mm}\end{array}$} & 3 & 6-pole & 0.00 & $1.00 \times 10^{-6}$ \\
\hline & 4 & 8-pole & 0.00 & $1.00 \times 10^{-6}$ \\
\hline & 5 & 10-pole & 0.00 & $1.00 \times 10^{-6}$ \\
\hline & 6 & 12-pole & $8.00 \times 10^{-6}$ & $1.00 \times 10^{-6}$ \\
\hline & 7 & 14-pole & 0.00 & $1.00 \times 10^{-6}$ \\
\hline & 8 & 16-pole & 0.00 & $1.00 \times 10^{-6}$ \\
\hline & 9 & 18-pole & 0.00 & $1.00 \times 10^{-6}$ \\
\hline & 10 & 20-pole & $-1.25 \times 10^{-7}$ & $1.00 \times 10^{-6}$ \\
\hline & 11 & 22-pole & 0.00 & $1.00 \times 10^{-6}$ \\
\hline & 12 & 24-pole & 0.00 & $1.00 \times 10^{-6}$ \\
\hline & 13 & 26-pole & 0.00 & $1.00 \times 10^{-6}$ \\
\hline & 14 & 28-pole & $-7.5 \times 10^{-9}$ & $1.00 \times 10^{-6}$ \\
\hline & 15 & 30-pole & 0.00 & $1.00 \times 10^{-6}$ \\
\hline & 16 & 32-pole & 0.00 & $1.00 \times 10^{-6}$ \\
\hline & 17 & 34-pole & 0.00 & $1.00 \times 10^{-6}$ \\
\hline & 18 & 36-pole & $-1.20 \times 10^{-6}$ & $1.00 \times 10^{-6}$ \\
\hline & 19 & 38-pole & 0.00 & $1.00 \times 10^{-6}$ \\
\hline \multirow{9}{*}{$\begin{array}{l}\text { Sextupole } B_{n} / B_{2} \\
\quad @ 13.5 \mathrm{~mm}\end{array}$} & 4 & 8-pole & 0.00 & $1.00 \times 10^{-6}$ \\
\hline & 5 & 10-pole & 0.00 & $1.00 \times 10^{-6}$ \\
\hline & 6 & 12-pole & 0.00 & $1.00 \times 10^{-6}$ \\
\hline & 7 & 14-pole & 0.00 & $1.00 \times 10^{-6}$ \\
\hline & 8 & 16-pole & 0.00 & $1.00 \times 10^{-6}$ \\
\hline & 9 & 18-pole & $-4.00 \times 10^{-6}$ & $1.00 \times 10^{-6}$ \\
\hline & 15 & 30-pole & $-1.00 \times 10^{-7}$ & $1.00 \times 10^{-6}$ \\
\hline & 21 & 42-pole & $1.30 \times 10^{-6}$ & $1.00 \times 10^{-6}$ \\
\hline & 27 & 54-pole & $-5.75 \times 10^{-7}$ & $1.00 \times 10^{-6}$ \\
\hline
\end{tabular}

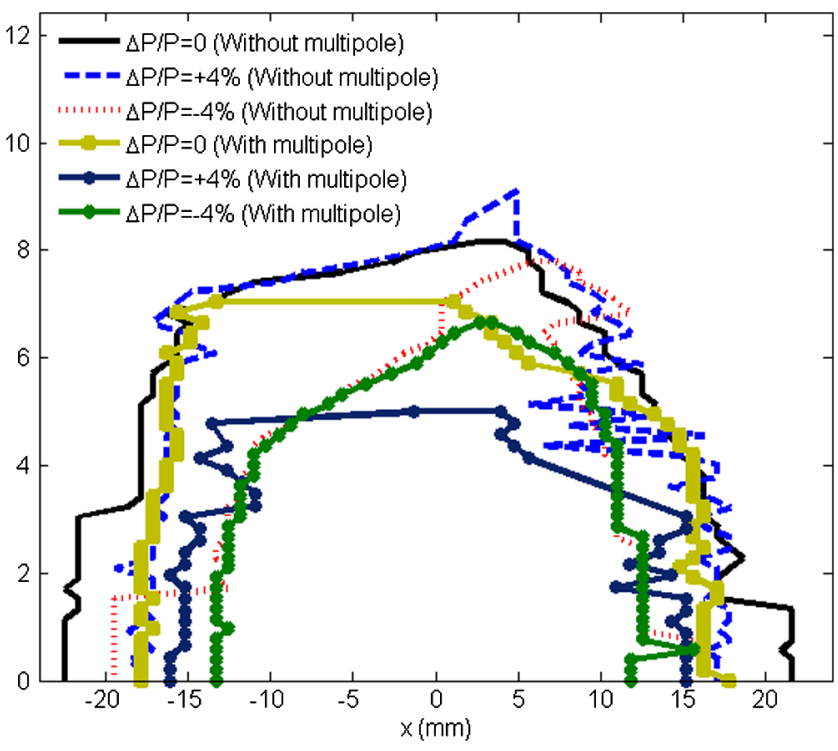

FIG. 8. The dynamic aperture at the center of the straight section. The multipole errors of the magnets are included.

additional windings in the sextupole magnets are employed and the singular value decomposition (SVD) method [25] is used for the orbit correction. The corrected closed orbits for 100 seeds of the random errors are shown in Fig. 9. The rms values of the corrected orbit are depicted in Fig. 10 and the corresponding strength of the corrector magnets is shown in Fig. 11. The results reveal that the closed orbit can be corrected to less than $\pm 60 \mu \mathrm{m}$ with this global correction scheme while the maximum strength of the correctors used for the orbit correction is $0.15 \mathrm{mrad}$.

\section{GOOD FIELD REGION}

The good field region (GFR) is evaluated from the off momentum deviated orbit, beam size, maximum distorted closed orbit, and the tolerance of beam offset as

$$
\begin{aligned}
\mathrm{GFR}_{i}= & \pm\left[a_{i} \times \eta_{i} \times \Delta+5 \sigma_{i}+b_{i} \times \mathrm{MAX} . \mathrm{COD}_{i}\right. \\
& \left.+ \text { tolerance of beam offset }\left.\right|_{i}\right]
\end{aligned}
$$

where the subscript $i$ indicates horizontal or vertical direction, $\eta$ and $\Delta$ represent dispersion function and energy

TABLE III. The utilized errors for the COD calculation. The errors have been cut off at 2 sigma.

\begin{tabular}{lcc}
\hline \hline Parameter & Unit & Value \\
\hline Transverse displacement of dipoles and sextupoles with respect to the girder & $\mu \mathrm{m}$ & 25 \\
Transverse displacement of quadrupoles and BPMs with respect to the girder & $\mu \mathrm{m}$ & 15 \\
Roll of dipoles, quadrupoles, sextupoles and BPMs with respect to the girder & $\mu \mathrm{rad}$ & 50 \\
Transverse displacement of girders & $\mu \mathrm{m}$ & 50 \\
Roll of the girder & $\mu \mathrm{rad}$ & 100 \\
Dipole fractional strength error & $\ldots$ & $5 \times 10^{-4}$ \\
Quadrupole fractional strength error & $\ldots$ & $1 \times 10^{-3}$ \\
Sextupole fractional strength error & $\ldots$ & $5 \times 10^{-3}$ \\
\hline \hline
\end{tabular}



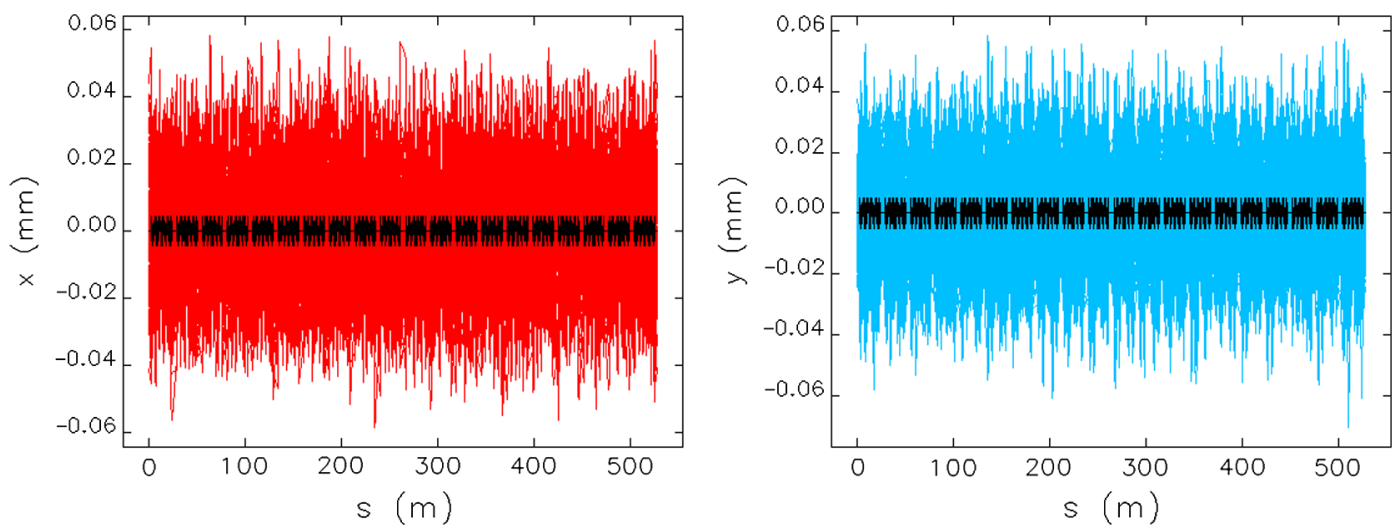

FIG. 9. Corrected closed orbits along the storage ring for 100 seeds of random errors: (left) horizontal orbit, (right) vertical orbit.
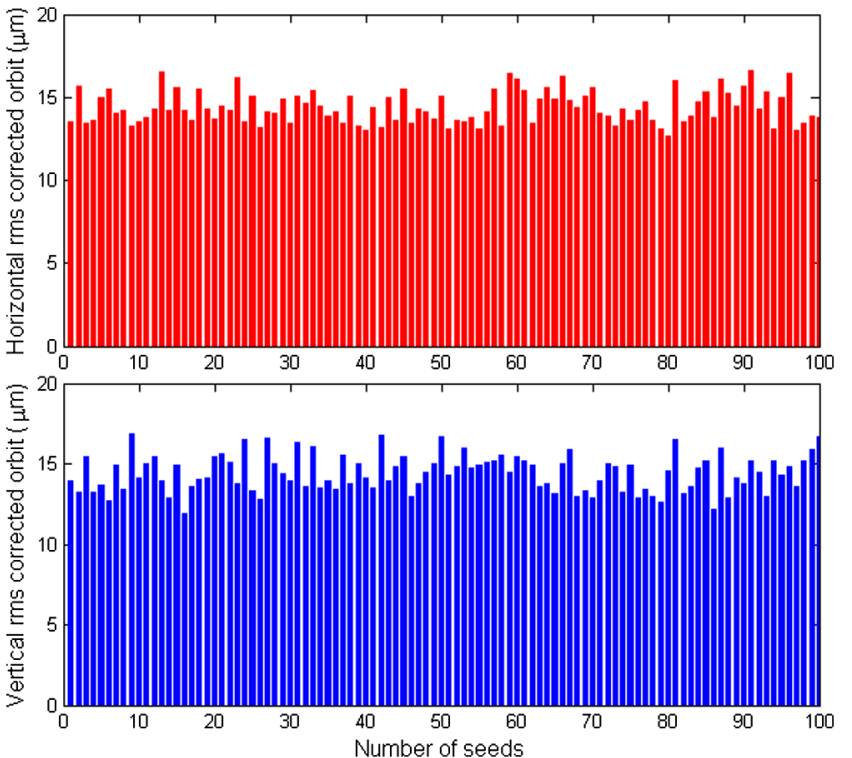

FIG. 10. The rms values of the corrected closed orbits for 100 seeds of random errors: (top) horizontal orbit, (bottom) vertical orbit.

error respectively. The coefficients $a$ and $b$ are used for safety to the orbit of off energy electrons and closed orbit respectively. In the ILSF lattice, we have utilized $a_{x}=a_{y}=2, \quad b_{x}=2.5, \quad b_{y}=2 \quad$ and considered $4 \mathrm{~mm} / 2 \mathrm{~mm}$ tolerances for beam centroid offset in the horizontal/vertical direction. The good field region in a super period of the storage ring is depicted in Fig. 12 which indicates horizontal/vertical GFR of $\pm 13.3 \mathrm{~mm} / \pm 11 \mathrm{~mm}$ in the magnets.

\section{SUPER-BEND MAGNET}

Due to using low field dipoles, the radiated beam critical energy is limited to $4.48 \mathrm{keV}$ at $3 \mathrm{GeV}$. This photon beam energy is useful for several experiments in the soft $\mathrm{x}$-ray region but it is low for the experiments which require high energy hard $\mathrm{x}$ ray. To reach super bright high energy
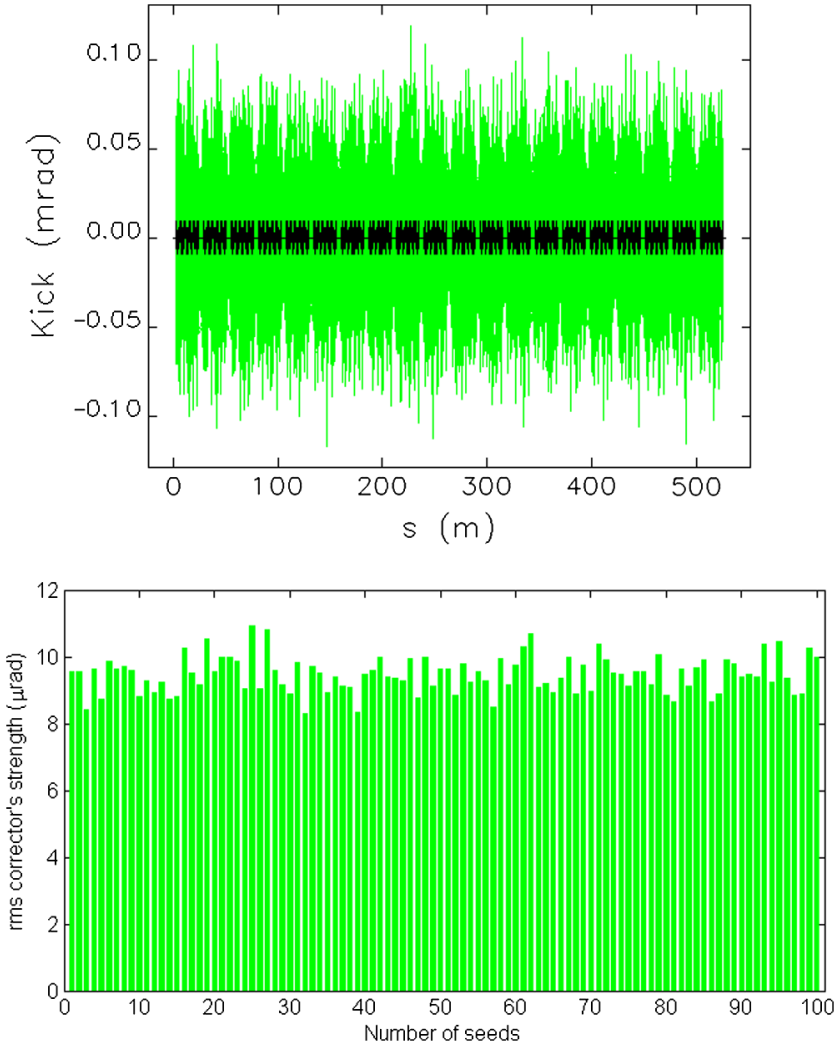

FIG. 11. (top) Strength of the corrector magnets for 100 seeds of random error. (bottom) The rms values of the corrector's strength versus seed number.

radiation from the dipoles, the central low field bending magnet (B3) in the lattice is replaced with a combination of a thin high field (HF) dipole magnet which is sandwiched between two low field (LF) dipoles. This replacement is shown in Fig. 13. With the use of such a super-bend magnet, the desired radiation would be achievable from the inserted high field section.

The longitudinal gradient provided with the super-bend magnet helps beam emittance reduction [26-30]. To simplify magnet design, avoiding a curve orbit dipole 
and easy fabrication procedure of this combination with home industry, the central dipole is split in three straight low field bending sections. All the bending sections are the same in the length $(28 \mathrm{~cm})$ and the deflection angle (1.2 degrees). The middle low field section is removable.
It can be taken out and replaced with the thin high field dipole, see Fig. 14. The natural emittance for a ring based on the 5BA lattice structure in the presence of high field inserted dipole with mentioned specifications is given by

$$
\varepsilon=\frac{C_{q} \gamma^{2}}{J_{x}} \cdot\left[\frac{2 N h_{0}^{3}\left[\int_{0}^{L_{0}}\left(H_{1}+H_{2}\right) \mathrm{ds}\right]+(N-n) h_{0}^{3} \int_{0}^{L_{0}} H_{3} \mathrm{ds}+2 n h_{0}^{3} \int_{0}^{\frac{L_{0}}{3}} H_{\mathrm{LF}} \mathrm{ds}+n\left(\frac{h_{0}}{\kappa}\right)^{3} \int_{0}^{\frac{\kappa L_{0}}{3}} H_{\mathrm{HF}} \mathrm{ds}}{4 N h_{0}^{2} L_{0}+(N-n) h_{0}^{2} L_{0}+2 n h_{0}^{2} \frac{L_{0}}{3}+n\left(\frac{h_{0}}{\kappa}\right)^{2} \frac{\kappa L_{0}}{3}}\right],
$$

where $C_{q}=3.84 \times 10^{-13}$ is the coefficient of quantum fluctuation for the electron, $\gamma$ is the relativistic factor, $J_{x}$ is the horizontal damping partition number, $N$ is the number of super period, $n$ is the number of high field inserted dipole, $L_{0}$ and $h_{0}$ are the length and the curvature of the low field dipoles (B1 or B2 or B3) in the bare lattice respectively. The $\kappa$ factor represents length ratio of high field (HF) and low field (LF) dipoles $\left(L_{H}=\kappa L_{0} / 3=\kappa L_{F}\right)$ and indicates how far the high field section can be extended.

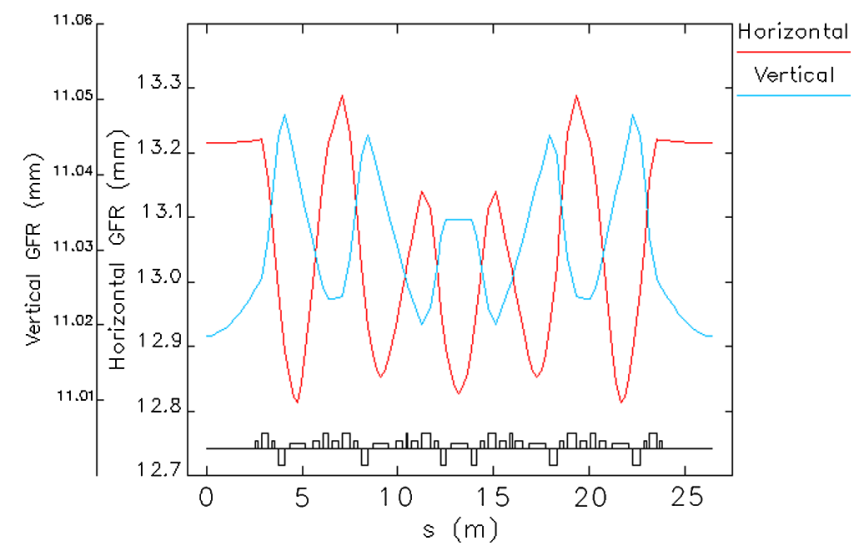

FIG. 12. The good field region in a super period of the storage ring. $\pm 4 \%$ energy error is assumed.
The beam emittance variation as a function of $\kappa$ factor for the different number of $n$ is displayed in Fig. 15. It reveals that the beam emittance lower than $0.45 \mathrm{~nm}$ rad would be achievable for the $\kappa=0.33$ when highest numbers of the high field sections are used. However, due to the saturation of locally available low carbon steel at $1.8 \mathrm{~T}$, the $\kappa$ factor is set to 0.43 [10] which indicates $12 \mathrm{~cm}$ for the length of high field inserted dipole.

\section{ILSF OPERATION SCENARIOS}

In the commissioning phase (phase 0), the total energy loss is only due to the synchrotron radiation from 100 low field dipoles. In the initial user mode (phase 1), we will employ four of the inserted high field dipoles with the field of $1.75 \mathrm{~T}$ and some commonly used IDs with different length that can be tailored to the specific requirements of the beam lines. The main parameters of the IDs are given in Table IV and Table V describes the ILSF operation scenarios. They indicate that one superconducting wiggler (SCW) magnet, two elliptical polarizing undulators (EPU) and three in-vacuum undulators (IVU) will be employed for the initial user mode of operation. The brilliance curves of the emitted radiation in the first user phase of operation are shown in Fig. 16.

The magnetic field of the IDs results the lattice beta beating and shrinkage of the dynamic aperture. Cure of the beta beating is done by the fine adjustment of four

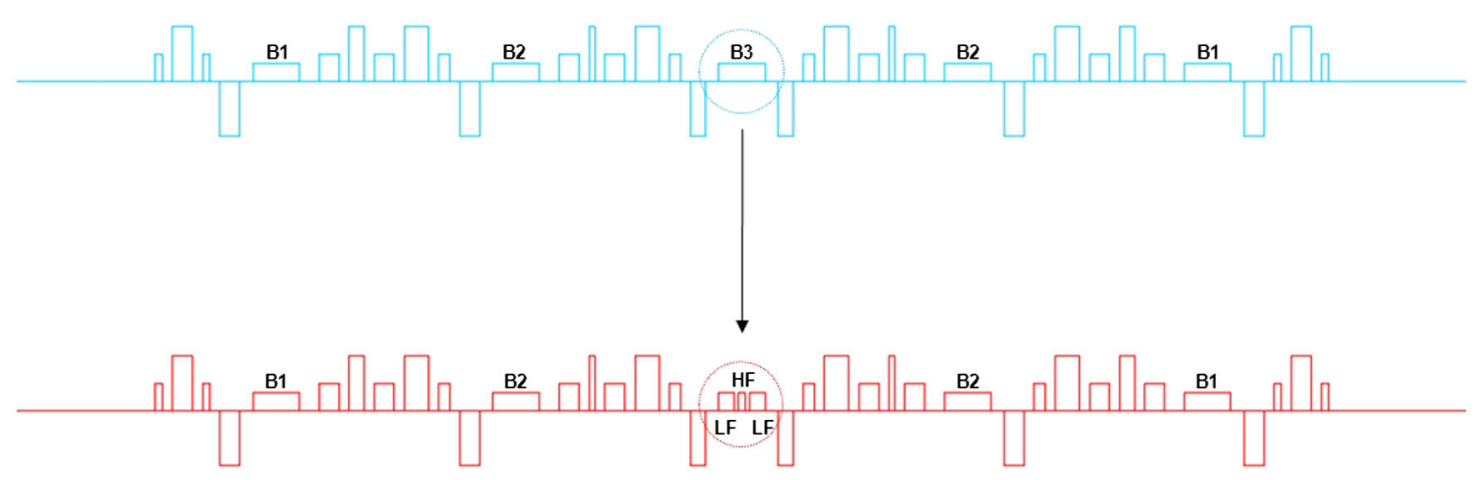

FIG. 13. The high field dipole magnet is sandwiched between two low field dipoles to radiate high energy super bright photons. This combination in the red lattice is remarked inside a dashed circle while the blue lattice represents the bare low field lattice. It should be noted that B1, B2 and B3 are the same in the specifications. 


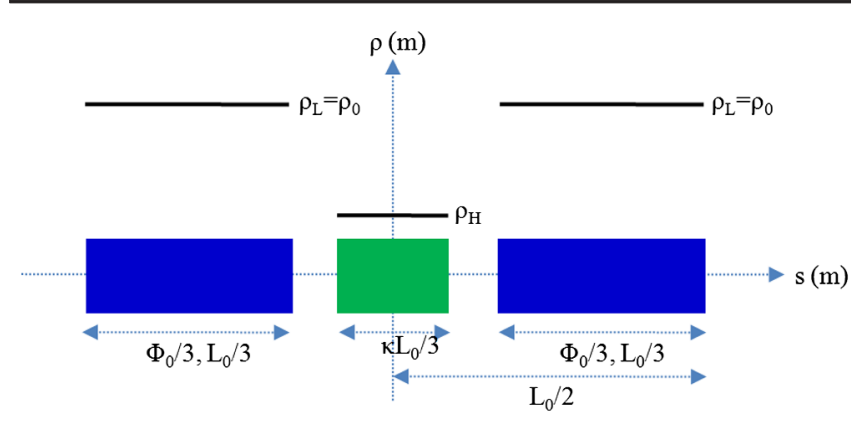

FIG. 14. The central low field dipole is split on three bending sections. The middle section (green color) is the high field inserted dipole which is sandwiched between two low field dipoles (blue color). The $\kappa$ factor can vary from 0 to 1 .

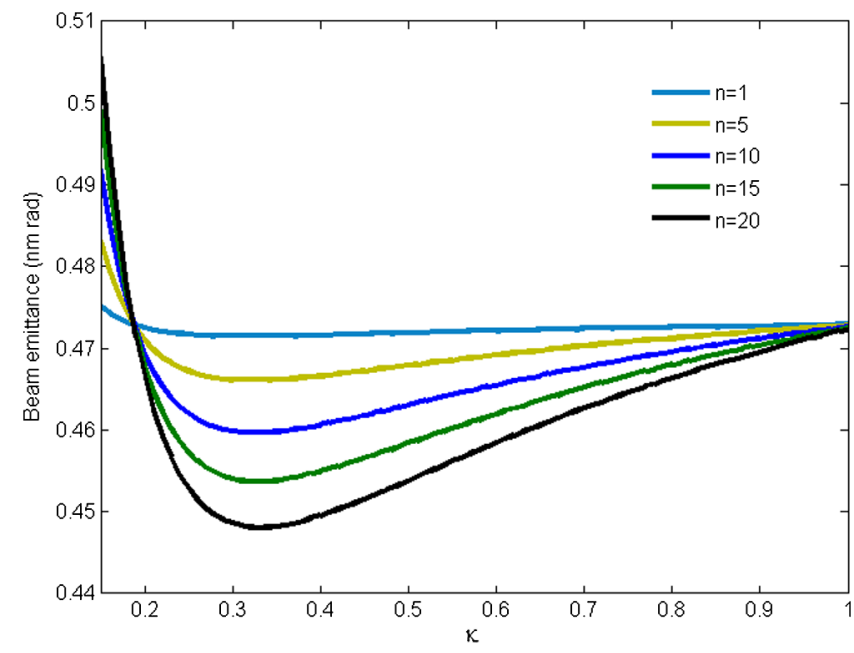

FIG. 15. Beam emittance as a function of $\kappa$ factor for the different number of the inserted high field section.

quadrupoles near to the IDs. The dynamic aperture of on/off momentum electrons in the presence of phase 1 IDs after 1000 turns tracking through the storage ring is depicted in Fig. 17. It reveals enough large physical stable area needed for the beam injection procedure.

In the final scenario of operation (phase 2) when more IDs will be installed in the ring operating at $400 \mathrm{~mA}$, we need to use higher rf voltage to compensate the higher energy loss per turn and install a third harmonic cavity for bunch lengthening and lifetime improvement.

\section{LIFETIME}

The major loss mechanisms in an electron storage ring are Touschek effect, elastic and inelastic scatterings against the residual gas molecules which are respectively called Coulomb and Bremsstrahlung scatterings [31]. The lattice energy acceptance for the realistic vacuum tube of the ILSF is depicted in Fig. 18. It reveals that the minimum lattice acceptance is more than $\pm 5 \%$ and choosing $2 \mathrm{MV}$ or larger as the total cavity gap voltage is adequate to obtain $\pm 4 \% \mathrm{rf}$ momentum acceptance which is crucial to achieve long Touschek lifetime in the storage ring.

However, due to the low value of beam emittance in the ILSF ring, the intrabeam scattering (IBS) effect must be studied on Touschek lifetime. The ELEGANT [13] simulation code is employed to calculate IBS effect and the results given in Table VI indicate $7.76 \%$ and $2.63 \%$ increase in beam emittance and beam energy spread respectively for the initial user mode. The filling factor of $80 \%$ in the initial user mode of operation is assumed which results 704 bunches each with the charge of $0.25 \mathrm{nC}$ in the ring. The Touschek lifetime as a function of the beam emittance is depicted in Fig. 19. As shown, the beam emittance in the bare lattice is setting almost in the minimum point of the black solid line curve. As the emittance of the storage ring is further increased by IBS effect, Touschek lifetime increases due to the beam size blowup in all dimensions. Adding further IDs and utilizing parasitic losses, the rf energy acceptance reduces which results lower Touschek lifetime.

In order to evaluate total beam lifetime, effects of the residual gas within the vacuum chamber must be considered. The Coulomb lifetime depends on the physical ring acceptance and residual gas composition. The physical ring acceptance is directly proportional to the square of vacuum chamber half gap $\left(g^{2}\right)$ and inversely to the beta function $(\beta)$. In our lattice, it limits horizontally to horizontal beta function $\left(\beta_{x}=18 \mathrm{~m}, g_{x}=14 \mathrm{~mm}\right)$ and vertically to the low gap of the in-vacuum undulator used in the initial user mode $\left(\beta_{y}=4 \mathrm{~m}, g_{y}=3 \mathrm{~mm}\right)$. The residual gases in the vacuum chamber are mainly hydrogen, oxygen, nitrogen

TABLE IV. Main specifications of the IDs in the first phase of operation where the beam current is $100 \mathrm{~mA}$.

\begin{tabular}{lccccc}
\hline \hline Parameters & Unit & Symbol & SCW & EPU & IVU \\
\hline Number of ID period & $\ldots$ & $N_{\mathrm{ID}}$ & 20 & 30 & 140 \\
Period length & $\mathrm{cm}$ & $\lambda_{\mathrm{ID}}$ & 6 & 8 & 1.6 \\
Magnetic field & $\mathrm{T}$ & $B_{y} / B_{x}$ & $3.500 / 0.000$ & $0.900 / 0.500$ & $0.850 / 0.000$ \\
Length of ID & $\mathrm{m}$ & $L_{\mathrm{ID}}$ & 1.200 & 2.400 & 2.240 \\
K parameter & $\ldots$ & $K_{y} / K_{x}$ & $19.614 / 0.000$ & $6.725 / 3.736$ & $1.270 / 0.000$ \\
Radiation loss & $\mathrm{keV}$ & $U_{r}$ & 83.789 & 14.500 & 9.225 \\
Power & $\mathrm{kW}$ & $P_{\mathrm{ID}}$ & 8.379 & 1.450 & 0.922 \\
\hline \hline
\end{tabular}


TABLE V. The operation scenarios for the ILSF storage ring. Beta beating is corrected by the nearest quadrupoles to the IDs. Maximum change in the gradient of the quadrupoles is $0.23 \mathrm{~T} / \mathrm{m}$.

\begin{tabular}{|c|c|c|c|}
\hline Parameters & Unit & Phase 0 (commissioning) & Phase 1 (initial user mode) \\
\hline Beam current & $\mathrm{mA}$ & 100 & 100 \\
\hline rfvoltage & MV & 2.000 & 2.000 \\
\hline Beam emittance & $\mathrm{nm} \mathrm{rad}$ & 0.476 & 0.412 \\
\hline Betatron tune, horizontal/vertical & $-/-$ & $43.283 / 14.255$ & $43.301 / 14.145$ \\
\hline Beam energy spread & $\cdots$ & $0.703 \times 10^{-3}$ & $0.835 \times 10^{-3}$ \\
\hline $\begin{array}{l}\text { Damping times, horizontal/vertical/ } \\
\text { longitudinal }\end{array}$ & $\mathrm{ms} / \mathrm{ms} / \mathrm{ms}$ & $19.709 / 19.716 / 9.859$ & $16.083 / 16.087 / 8.045$ \\
\hline Coupling $(\%)$ & $\cdots$ & 1 & 1 \\
\hline Bunch length & $\mathrm{mm}$ & 2.438 & 2.926 \\
\hline Momentum acceptance $(\%)$ & $\cdots$ & 4.339 & 4.054 \\
\hline IDs & $\cdots$ & $\ldots$ & $4 \mathrm{HF}+\mathrm{SCW}+3 \mathrm{IVU}+2 \mathrm{EPU}$ \\
\hline Radiation loss per turn & $\mathrm{keV}$ & 535.975 & 656.855 \\
\hline Parasitic loss & $\mathrm{keV}$ & 30 & 30 \\
\hline
\end{tabular}

and few ratios of the organic compounds. Because of the high atomic number of $N_{2}$ which results strong effects on the lifetime, the nitrogen gas at the temperature of $300 \mathrm{~K}$ within the vacuum chamber is assumed in the calculation. The Bremsstrahlung lifetime is given by momentum acceptance which is $4.05 \%$ for $2 \mathrm{MV}$ rf gap voltage. The total lifetime versus gas pressure within the pipe is given in Fig. 20. It reveals 12.79 hours storage ring total lifetime at the typical pressure of 1.5 nTorr.

\section{INJECTION}

For the stable operation, minimization of disruption of the storage ring and to reach top-up operation mode, the injection procedure must be very efficient in such a way that no electrons should be lost. That would protect problems due to the radiation and would provide very

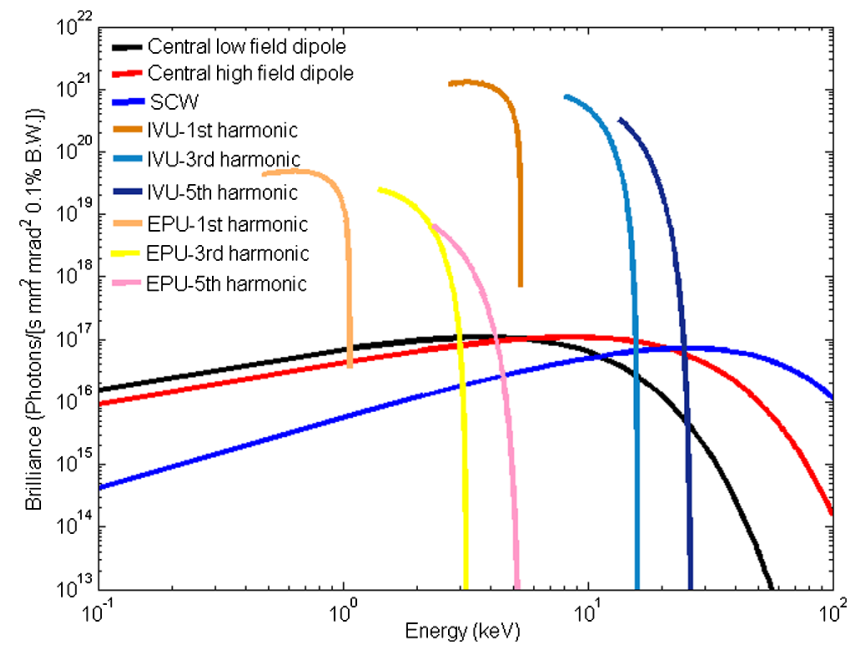

FIG. 16. On axis brilliance of the synchrotron radiation in the ILSF first user phase of operation. stable x-ray beam for the users without any interruptions during their experiments. A conventional injection scheme is used for this propose. The main body of the designed injection system is composed of one septum magnet placed at the end of the booster to storage ring transfer line and four kicker magnets which are distributed symmetrically in one $5.11 \mathrm{~m}$ straight section of the storage ring. Length of the each kicker is $500 \mathrm{~mm}$ and can provide a kick angle of $10 \mathrm{mrad}$, see Fig. 21.

The kicker magnets produce a smooth orbit bump near the septum magnet to capture the injected electron beam brings by the septum magnet into the vacuum chamber of the storage ring. The height of the orbit bump for different kicker amplitude is displayed in Fig. 21. As an advantage of this injection scheme, the local orbit bump only depends on the strength of kickers. This is because no storage ring magnets appeared in between the injection kickers and

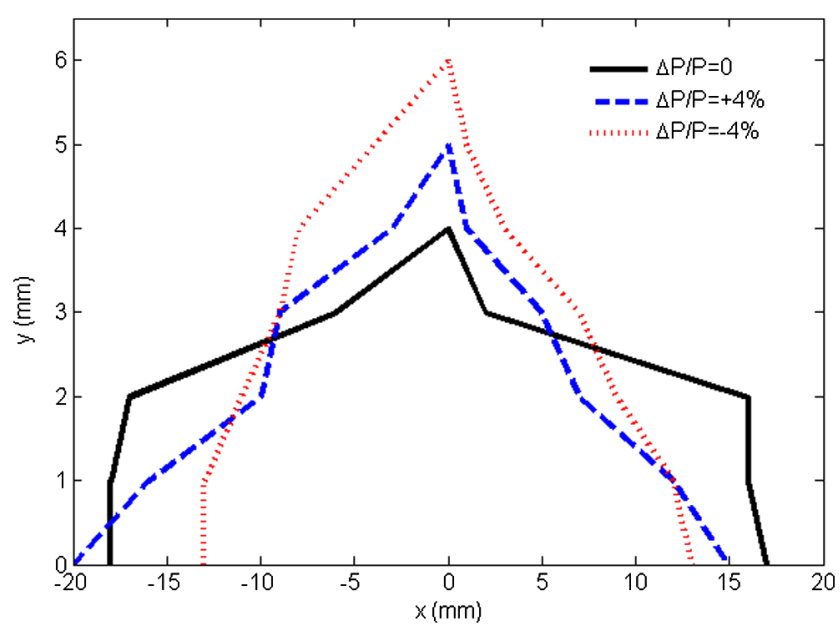

FIG. 17. The dynamic aperture in the presence of phase 1 IDs. The multipole errors have been included in the particle tracking. 


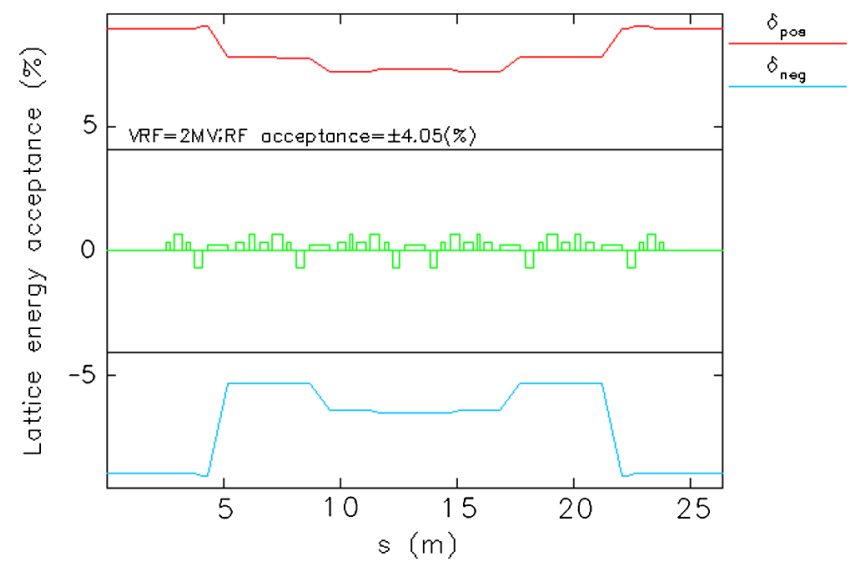

FIG. 18. Lattice momentum acceptance in a super period of the storage ring. The red and blue curves represent positive and negative energy acceptances. $\pm 4.05 \%$ rf acceptance is specified with straight black lines.

strength of the kickers directly affects height of the orbit bump. The separations between the stored, bumped and injected beams are depicted in Fig. 22. It indicates that the injected beam has a distance of $18 \mathrm{~mm}$ from the main orbit and width of septum magnets is assumed to be $3 \mathrm{~mm}$. The height of the bumped orbit is $10 \mathrm{~mm}$ and distance between the beams and the septum magnet is utilized to be long enough to prevent scraping of the electrons. In order to study performance of the designed injection system, the ELEGANT [13] code as a 6D particle tracking software has been used and 1000 electrons in a Gaussian distribution per bunch are tracked through the ring. Since the injection system is located in a straight section, perfect orbit bump would exist if the kickers operate without any error. However, the errors have to be included to observe real motion of the electron beam. The errors of the injected beam influence efficiency of the injection procedure but do not have any effect into the stored beam and thus they are not the main concern here. A mismatch injected beam is an example of this type of error. Moreover, any errors in the storage ring element can affect the injection efficiency but our main attention here is the errors due to the injection system elements in particular kicker magnets. Since the

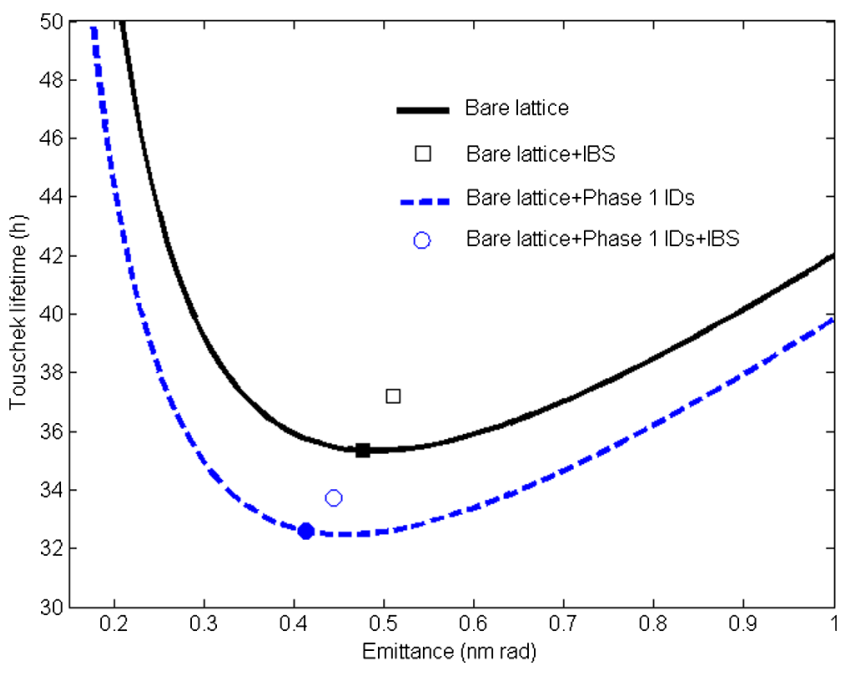

FIG. 19. Touschek lifetime as a function of beam emittance for both commissioning and initial user phases. The rf gap voltage is 2 MV. The effect of IBS is neglected in the filled circle and rectangle. The beam energy spread is kept constant at each curve.

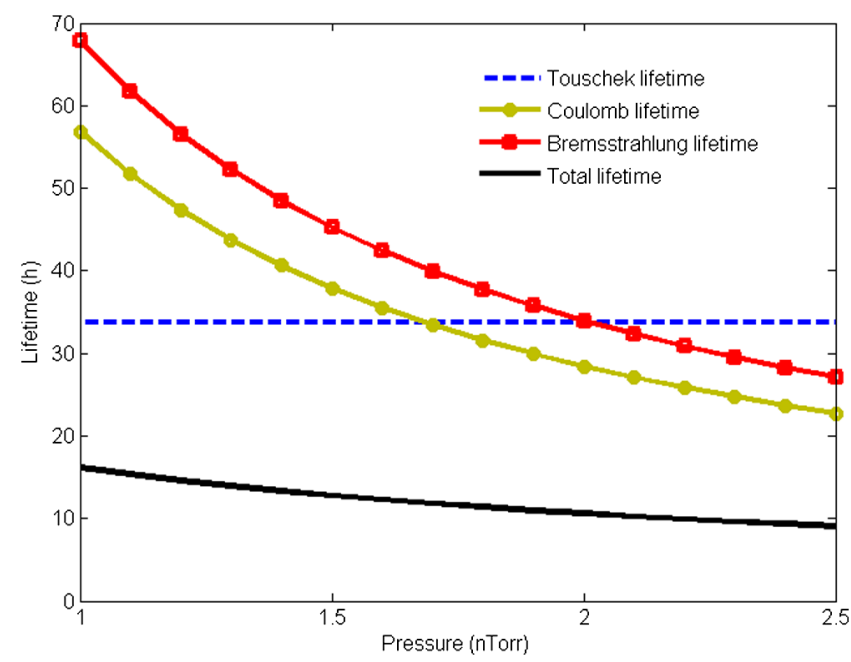

FIG. 20. Lifetime in the storage ring versus pressure within the pipe. Momentum acceptance is $4.05 \%$.

TABLE VI. Equilibrium horizontal beam emittance, beam energy spread and Touschek lifetime calculated with ELEGANT [13] code with and without IBS effect.

\begin{tabular}{lccc}
\hline \hline & Parameters & Without IBS & With IBS \\
\hline \multirow{2}{*}{ Phase 0 (commissioning) } & Beam emittance (nm rad) & 0.476 & 0.511 \\
& Beam energy spread & $0.703 \times 10^{-3}$ & $0.732 \times 10^{-3}$ \\
& Touschek lifetime (h) & 35.338 & 37.220 \\
\hline & Beam emittance (nm rad) & 0.412 & 0.444 \\
Phase 1 (initial user mode) & Beam energy spread & $0.835 \times 10^{-3}$ & $0.857 \times 10^{-3}$ \\
& Touschek lifetime (h) & 32.589 & 33.703 \\
\hline \hline
\end{tabular}




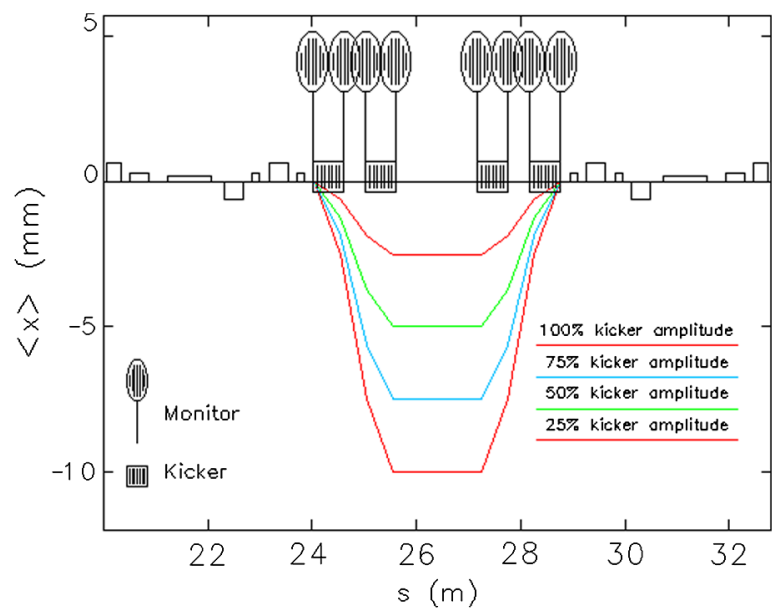

FIG. 21. The horizontal trajectory of the stored electron beam centroid during passing through the kicker magnets. Several monitors have been distributed before and after each injection kicker and after the septum magnet to observe phase space coordinate of the electron beam.

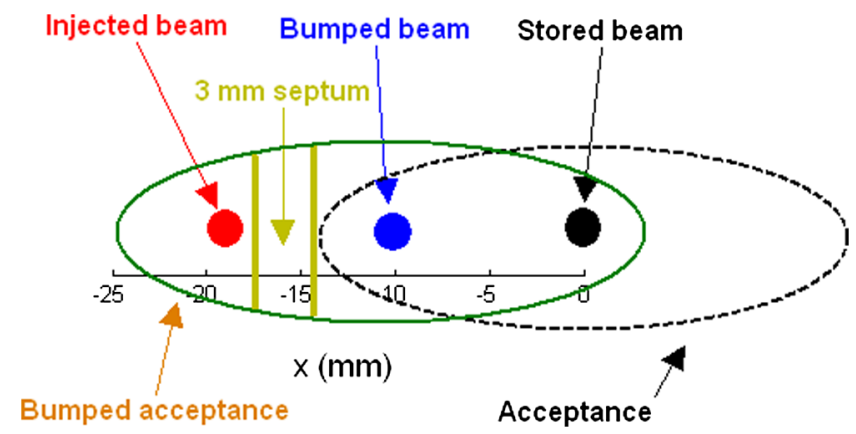

FIG. 22. Schematic layout of the separations between the stored, bumped and injected beams.

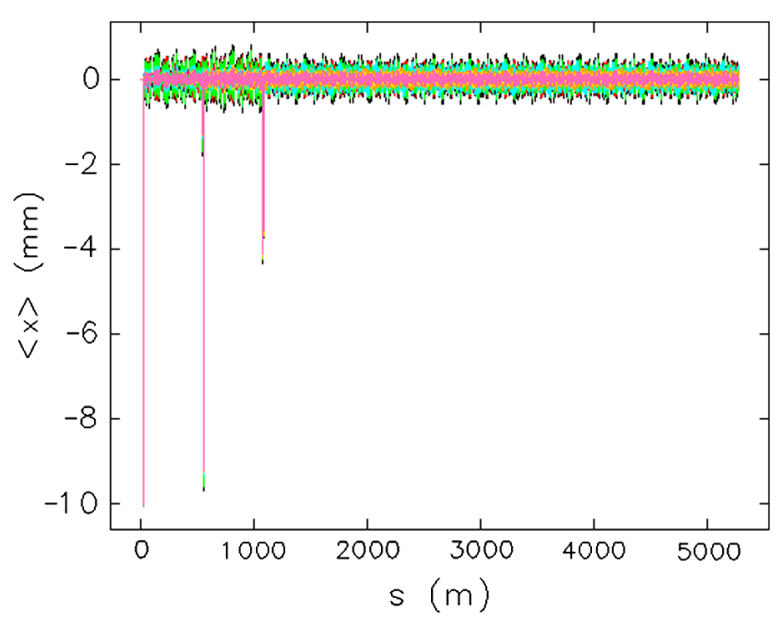

FIG. 23. The horizontal trajectory of the stored beam centroid during ten turns passing through the low field ring. The errors of the injection kickers have been included. Colors represent the number of the error seeds.
TABLE VII. Main parameters of the injected beam.

\begin{tabular}{lccc}
\hline \hline Parameter & Symbol & Unit & Value \\
\hline Emittance & $\varepsilon$ & $\mathrm{nm} \mathrm{rad}$ & 3.503 \\
Energy spread & $\delta$ & $-/-$ & $8.460 \times 10^{-4}$ \\
Bunch length & $\sigma_{l}$ & $\mathrm{~mm}$ & 12.500 \\
Coupling & $\cdots$ & $\%$ & 10 \\
\hline \hline
\end{tabular}

kickers will have separate power supply for the operation, they will not sense any correlation effect. The utilized errors of the kickers in the simulation are as follows: rms peak field error of $0.1 \times 10^{-4}$, the rms time error of $1 \mathrm{~ns}$, the rms roll of the kickers of $50 \mu \mathrm{rad}$ and the transverse misalignment of $50 \mu \mathrm{m}$.

\section{A. Tracking of stored beam}

The horizontal trajectory of the stored beam centroid after passing ten turns through the ring is shown in Fig. 23. It shows different height of orbit bump due to the different strength of the kickers at each turn. After two turns passing through the storage ring, the kickers would be switched off and thus the electrons will move in the main ideal orbit. As shown, the errors disturb the perfect bump and a residual oscillation below $\pm 1 \mathrm{~mm}$ appeared in which, after the few damping times, it will be damped by the synchrotron radiation.

\section{B. Tracking of injected beam}

In order to observe performance of the injection system, the matched electron beam with a horizontal displacement of $18 \mathrm{~mm}$ has been injected into the storage ring. Main parameters of the injected beam are given in Table VII. Tracking results are displayed in Fig. 24. After ten turns

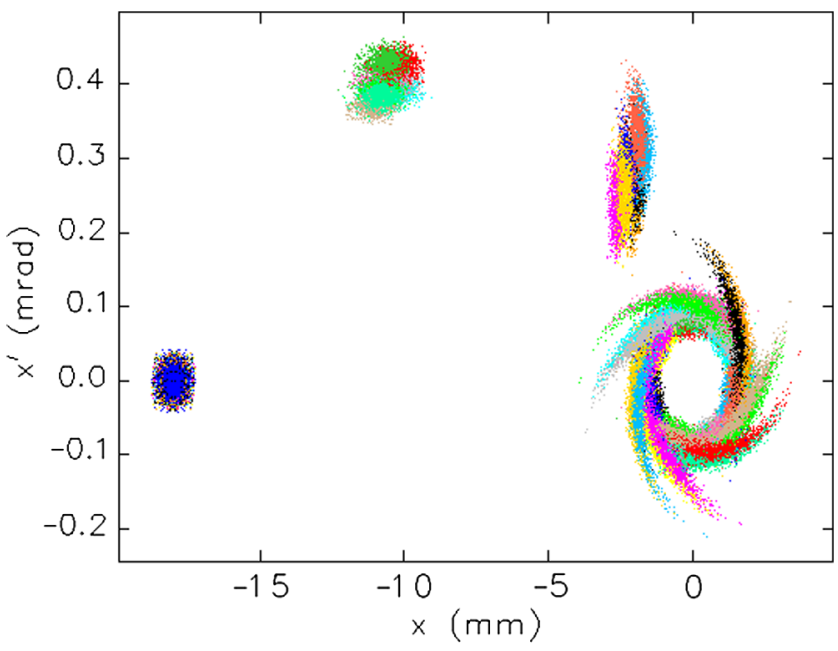

FIG. 24. Phase space coordinate of the matched injected beam during ten turns passing through the low field ring. Errors of the injection kickers have been included. Colors indicate the number of turns for different seeds. 
passing through the ring, the injected beam is trapped in acceptance angle smaller than $\pm 0.2 \mathrm{mrad}$. No loss of the electrons in the simulation indicates very efficient injection procedure.

\section{CONCLUSIONS AND OUTLOOK}

The ILSF storage ring has been designed based on a fivebend achromat lattice. Due to the local availability of the low carbon steel, the lattice has been designed based on the use of well-known relaxed weak magnets which leads lower fabrication cost of the magnets and lower operation cost in comparison with the high field lattice. The quadrupole and sextupole magnets have been designed with small aperture to provide the required strong field strength. It results in significant impact in cost reduction of the vacuum system. Circumference of the storage ring is $528 \mathrm{~m}$ and the beam emittance of $0.48 \mathrm{~nm}$ rad is obtained in the bare lattice. Fine optimization of the sextupole magnets results in large dynamic aperture for our compact lattice which is one of the main challenges in design of the ultralow emittance rings. The global orbit correction scheme is foreseen with use of additional windings in the sextupole magnets. The BPMs will be placed between these sextupoles and the adjacent quadrupoles. Fast orbit correction scheme is under investigation to fulfill good beam stability below than $1 \mu \mathrm{m}$. Ground vibration effects which cause magnets movement and result orbit stability are under study. Production of very bright high energy radiation with the use of a super-bend magnet and its corresponding effects on the beam parameters have been discussed. The super-bend magnet has been physically designed and its mechanical drawings have been prepared. It is now ready for fabrication as a prototype in the ILSF. Lifetime calculation including IBS effect has been explained. A conventional injection scheme is introduced for the injection into the ILSF ring and the use of pulsed multipole magnet as an alternative method is under study.

\section{ACKNOWLEDGMENTS}

The authors would like to especially thank $\mathrm{H}$. Wiedemann for his several helpful comments on this research. We also appreciate D. Einfeld, A. Wrulich and E. Weihreter for their kind help. This paper is supported by Iranian Light Source Facility.

[1] D. Einfeld, S. S. Hasnain, Z. Sayers, H. Schopper, and H. Winick, SESAME, a third generation synchrotron light source for the Middle East region, Radiat. Phys. Chem. 71, 693 (2004).

[2] A. K. Çiftçi, R. Çiftçi, H. Yıldız, and K. Zengin, Beam dynamics issues and synchrotron radiation on TAC-SR, Nucl. Instrum. Methods Phys. Res., Sect. A 675, 34 (2012).
[3] Candle light source conceptual design report, http://www .candle.am/TDA/index.htm.

[4] J. Rahighi, Proposal for a 3rd generation national Iranian synchrotron light source, in Proceedings of the International Particle Accelerator Conference, Kyoto, Japan (ICR, Kyoto, 2010), pp. 2532-2534; http://ilsf.ipm.ac.ir/.

[5] J. Rahighi et al., Third generation light source project in Iran, in Proceedings of the 2nd International Particle Accelerator Conference, San Sebastian, Spain (EPS-AG, Spain, 2011), pp. 2954-2956.

[6] H. Ghasem, D. Einfeld, F. Saeidi, and E. Ahmadi, Lattice candidates for the ILSF storage ring, in Proceedings of the 2nd International Particle Accelerator Conference, San Sebastian, Spain, (Ref. [5]), pp. 2957-2959.

[7] H. Ghasem, F. Saeidi, and E. Ahmadi, Low field low emittance lattice for the storage ring of Iranian Light Source Facility, JINST, 8, P02023 (2013).

[8] H. Ghasem, F. Saeidi, and E. Ahmadi, Lattice design history of the Iranian Light Source Facility storage ring, in Proceedings of the International Particle Accelerator Conference, Dresden, Germany (EPS-AG, Dresden, 2014), pp. 249-251.

[9] H. Ghasem, F. Saeidi, and E. Ahmadi, High field or low field lattice for the storage ring of Iranian Light Source Facility?, ILSF Beam Dynamic Group Internal Report No. ILSF-A-SR-SR-2013-01-02, 2013.

[10] A. Shahveh et al., Identification of magnetic field material for the ILSF magnets, ILSF Magnet Internal Report No. ILSF-MG-TN-RE17-00-20140501, 2014.

[11] A. Shahveh et al., Magnetic measurement report for $\mathrm{H}$ type prototype dipole, ILSF Magnet Internal Report No. ILSFMG-TN-RE07-01-20130701, 2013.

[12] A. Streun, OPA Lattice Design Code, https://ados.web.psi .ch/opa/.

[13] M. Borland, Elegant: a flexible sdds-compliant code for accelerator simulation, Advanced Photon Source, Argonne National Laboratory, USA, Report No. LS-287, 2000.

[14] Methodical Accelerator Design webpage.

[15] J. Payet, the upgraded version from the BETA developed by J. L. Farvaque, J. L. Laclare, and A. Ropert, Report No. BETA-LNS-V 7.80.

[16] T. Tanaka and H. Kitamura, SPECTRA: a synchrotron radiation calculation code, J. Synchrotron Radiat. 8, 1221 (2001).

[17] S. Ozaki, J. Bengtsson, S. L. Kramer, S. Krinsky, and V. N. Litvinenko, Philosophy for NSLS-II design with subnanometer horizontal emittance, in Proceedings of the 22nd Particle Accelerator Conference, Albuquerque, New Mexico (IEEE, New York, 2007), pp. 77-79.

[18] A. Xiao, M. Borland, and V. Sajaev, A 1-nm emittance lattice for the Advanced Photon Source storage ring, in Proceedings of the 22nd Particle Accelerator Conference, Albuquerque, New Mexico (Ref. [17]), pp. 3447-3449.

[19] Y. Papaphilippou, A. Ropert, P. Elleaume, and L. Farvacque, Lattice upgrade options for the ESRF storage ring, in Proceedings of the 21st Particle Accelerator Conference, Knoxville, Tennessee (IEEE, Piscataway, NJ, 2005), pp. 1-3.

[20] J.-L. Revol et al., ESRF Operation and upgrade status, in Proceedings of International Particle Accelerator 
Conference (2013), Shanghai, China (EPS-AG, Shanghai, 2013), pp. 82-84.

[21] L. Emery, Overview of advance photon source multi-bend achromat upgrade proposal, 4th Low Emittance Rings Workshop, Frascati, Italy, 2014, INFN-LNF.

[22] Sirius Detailed Design Report, 2014.

[23] S. C. Leemann, A. Andersson, M. Eriksson, L.-J. Lindgren, E. Wallen, J. Bengtsson, and A. Streun, Beam dynamics and expected performance of Swedens new storage-ring light source: MAX IV, Phys. Rev. ST Accel. Beams 12, 120701 (2009).

[24] V. Moradi et al., ILSF mechanics internal report, 2014.

[25] Y. Chung, G. Decker, and K. Evans, Closed orbit correction using singular value decomposition of the response matrix (Argonne National Laboratory, IL, 1993).

[26] Y. Papaphilippou and P. Elleaume, Analytical considerations for reducing the effective emittance with variable dipole field strengths, in Proceedings of the 21st Particle Accelerator Conference, Knoxville, Tennessee (Ref. [19]), pp. 1-3.
[27] R. Nagaoka and A. Wrulich, Emittance minimization with longitudinal dipole field variation, Nucl. Instrum. Methods Phys. Res., Sect. A 575, 292 (2007).

[28] J. Guo and T. Raubenheimer, Low Emittance $e^{-} / e^{+}$ storage ring using bending magnets with longitudinal gradient, in Proceedings of the 8th European Particle Accelerator Conference, Paris, 2002 (EPS-AG, CERN, Geneva, 2002), pp. 1-3.

[29] S. Papadopoulou and Y. Papaphilippou, Analytical considerations for reducing the emittance with longitudinally variable bends, 4th Low Emittance Rings Workshop, Frascati, Italy, 2014, INFN-LNF.

[30] Andreas Streun, Longitudinal gradient super-bends, and anti-bends for compact low emittance light source lattices, 4th Low Emittance Rings Workshop, Frascati, Italy, 2014, INFN-LNF.

[31] H. Wiedemann, Particle Accelerator Physics, 3rd ed. (Springer-Verlag, Berlin, 2007). 